\title{
EVALUATION OF AODV AND DYMO ROUTING PROTOCOL USING GENERIC, MICAZ AND MICAMOTES ENERGY CONSERVATION MODELS IN AWSN WITH STATIC AND MOBILE SCENARIO
}

\author{
SURESH KUMAR*1, KIRAN DHULL*, DEEPAK SHARMA*, ARORA PAYAL*AND SANDEEP DAHIYA ${ }^{\dagger}$
}

\begin{abstract}
Adhoc Wireless Sensor Networks (AWSN's) have found an increasing utility in various applications. In AWSN, sensor nodes are arranged in a topology which is suitable to the requirement of the nature of task. As the sensors are not connected in a fixed manner, their networking, location and functioning keeps on changing based on the situation due to which these are called Adhoc networks. The biggest challenge is to keep the sensors working for longer time by conserving their energy. Therefore, a suitable routing protocol needs to be selected to meet the energy conservation requirement at different nodes. In the present paper, we have evaluated the three energy conservation models i.e. Generic, Micaz and Micamotes for Ad-Hoc On demand Distance Vector (AODV) and Dynamic MANET On Demand (DYMO) routing protocol. The evaluation is carried out using the parameter metrices: Average End-to-End Delay (AEED), Throughput, Energy consumed in Transmit mode and Receive mode. Based on the simulation results, it has been observed that Micamotes energy model using AODV routing protocol performs better in terms of energy consumption upto $42.99 \%$ and $29.90 \%$ in transmit and up to $59.24 \%$ and $33.96 \%$ in receive mode respectively as compared to Generic and Micaz energy model.
\end{abstract}

Key words: WSN, Ad-hoc Wireless Sensor Network (AWSN), AODV, Mobile Ad-hoc Networks (MANETS), RREQ, RREP, RERR, DYMO, AEED, Total Packet Received (TPR), Packet Delivery Ratio (PDR), Optimized Link State Routing(OLSR)

AMS subject classifications. 68M10,68M20,60K05, 60K25

1. Introduction. AWSN consists of independent nodes that communicate with each other by preserving connectivity in a distributed manner and creating a multi hop radio system. Wireless communication ability is present in every sensor node. They also have intelligence for processing of signal and data networking. They draw energy from batteries for communication between the sensors and the network. While communicating with other sensors, the energy consumed is higher [1].

Deployment of AWSN's is not on a large scale as the research in this field is by and large simulation based. Among other simulation parameters, the mobility model in AWSN plays a vital role to evaluate the performance of the protocol. AWSN's are joint arrangement of mobile nodes without the support of any central node that can communicate with one another. It gives the realistic and efficient use of radio communication channel and multi hop radio relaying. End user controls this network with the enhancement technology rather than a single authority and can be used for particularly sensitive applications. In AWSN's, node mobility is a major problem because of the ad-hoc features like restricted bandwidth, vibrant network topology, shared medium, security and multi hop character etc. [2]. Seamless mobility is an efficient method of mobility management in ad-hoc networks which offers simple access and efficient communication between nodes present in the arrangement.

Simulation is one of the most significant method for testing the features of ad-hoc networking protocol which gives various benefits like parameters isolation, repeatable scenarios and discovery of a several metrics. Topology and movement of the nodes are the important issues in evaluating the performance of network protocol. The mobility model defines the status of the nodes which are moving in a position on being dispersed initially inside the network. Accurate performance cannot be obtained by simulation results that are achieved with idealistic movement models,since nodes mobility directly distracts the performance of the protocol and for AWSN, idealistic movement scenarios cannot be obtained by the majority of existing mobility models[3]. The architecture of AWSN is depicted in figure 1.1. WSN's are deployed in volatile and unfriendly environments where deployment using wired networks is not possible. In case of forests, it is difficult to go down and setup a wired network. Therefore, the sensor nodes are made to fall from the air. Scalability is another merit of WSN which allows its use in Structural Health Monitoring, as the fixed and dense deployment in wired mode is

*Department of Electronics and Communication Engineering, University Institute of Engineering and Technology, Maharshi Dayanand University, Rohtak, Haryana, India (sureshvashist.uiet.ece@mdurohtak.ac.in,, kirandhull19@gmail.com, d.29deepak@gmail.com payalarora325@gmail.com).

${ }^{\dagger}$ BPS Mahila Vishwavidayala, Khanpur, Haryana, India (sandydahiya2001@yahoo.com). 


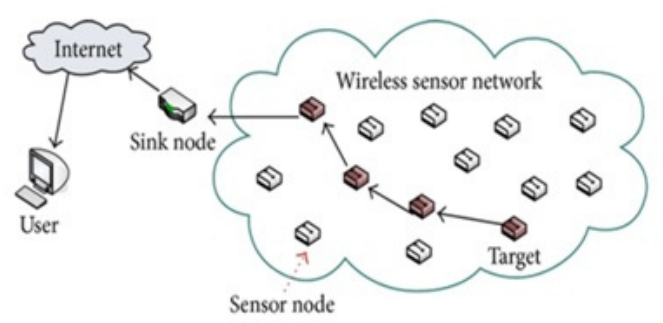

FIG. 1.1. Architecture of AWSN

required which is very expensive. However, WSNs can be deployed with least efforts and developed on a large scale according to the application. These can be networked easily and accessed with the remote area.

Three topologies (Mesh, Star and Tree) can be used for designing of the WSN's. These are economical,consist of little power with various nodes and at the same time can be maintained easily. Lesser energy computation capabilities and communication assets are the main disadvantage of WSN. In spite of these, it also has less storage space, low battery power and bandwidth and is prone to the attacks. Despite of these weaknesses, WSN's are broadly used and hence it may be rightly called as backbone of "Internet of Things". AWSN's are basically used in networks such as community and home network, vehicle and emergency response networks.

The other characteristics of AWSN's are unlicensed frequency spectrum, less cost of infrastructure instalment, sudden allocation of data around the sender, flexibility and mobility enhancement and capable of bringing up and tearing down in a very small time frame. Due to multi-hop support, communication beyond the Line of Sight (LOS) is possible at high frequencies, which diminishes the consumption of power of wireless devices. The Section 2 discusses the various routing protocols, Section 3 contains the recent related work reported in literature, the proposed work and simulation setup is described in Section 4. Section 5 covers the simulation results with discussion and Section 6 concludes the paper.

\section{Routing Protocols and Energy Models.}

2.1. AODV. AODV is one of the routing protocols, where every mobile host acts as a dedicated router. Concept of Bellman ford distance vector algorithm is mainly expanded by AODV in a virtual way. It was particularly considered for dynamic WSNs. It becomes the virtual dynamic WSN's, when unpredictable topology changes take place in WSN due to failure of nodes. To develop the ad-hoc network, AODV algorithm is fairly appropriate for dynamic self-starting network as required by users. It includes a series number and one entry per table which is exactly same as the conventional approach of routing to retain the routing data up to date. It is a reactive routing protocol where routes are created in case of requirements. It ensures loop-free routes in the altered condition and also in case of repairing broken links where any node ought to be treated as dead node if currently not in use.

The demand of bandwidth existing to the mobile nodes is significantly less because the protocol does not practice any type of universal cyclic advertisement. Symmetric links are used by AODV among the adjusting nodes and it does not track the path between nodes, if one of the nodes cannot attend the other one. Nodes neither keep any routing data nor contribute in any periodic exchange of routing table and also do not lie on dynamic path. It comprises the conventional concept of routing table that includes the factors like: address of next hop, routing information, a series number and node handling, since the node retain the specific time spam after that its entrance must be discarded. It is notified by the adjusting node if the link is not working. In AODV, two series are used for obtaining the routing mainly (i) Query (ii) Reply with the control requests i.e. RREQ, RREP, RERR and HELLO message. While execution, RREQ message broadcasted by a node to the other node, thereafter the RREP message comes as unicast. The neighbouring nodes observe the RERR message only if the link is disconnected. For estimation and detection of the links, the HELLO message is used among the nodes. Figure 2.1 depicts the AODV Routing protocol. 


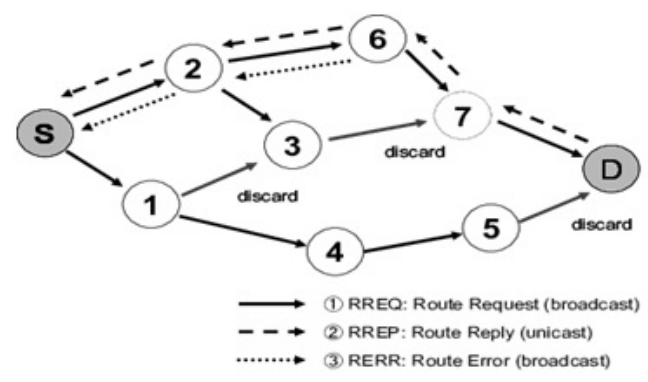

FIG. 2.1. AODV Routing Protocol

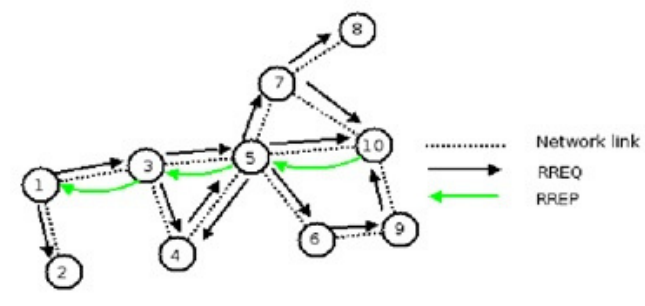

FIG. 2.2. DYMO routing protocol

2.2. DYMO. For multi-hop networks, DYMO is one of the effortless and high-speed routing protocols. It determines the routes based on the demand request and gives improved coverage for dynamic topologies inside the networks. To find out the route, data packet was transmitted by the source with RREQ message same as the AODV protocol. After receiving the RREQ message, DYMO waits and look for assignment of a route and it may issue another RREQ, if route is not assigned within waiting period. For minimising the obstruction, exponential back off method is used in the network. Figure 2.2 depicts the DYMO routing protocol network. When data packets have not been delivered then RERR message is issued to the destination due to absence of route. Small state information such as the active source and destination is retained in the DYMO router because the applicable devices like WSN have less memory [3].

Various Energy Models used for energy conservation are Generic, Micaz and Micamotes. To minimise the consumption of energy, Generic energy model is used to reduce the task which are completed by the sensors and their linked networks. Various approaches have to be used to replenish the energy capacity of sensor by constructing apparatus and mechanism for extra energy harvesting. The low power WSNs, Micamotes energy model used by researchers are second generation motes. It works at frequency $4 \mathrm{MHz}$ and processor used by it is Atrnel ATmega $128 \mathrm{~L}$ which is an 8 bit microcontroller and has $128 \mathrm{~Kb}$ flash memories to store the mote program. It consumes $8 \mathrm{~A}$ and $15 \mathrm{~A}$ current in running mode and sleep mode respectively. Two AA batteries are used by Micamotes which can run for more than a year. Micaz is a third generation tool used for improvement of WSNs which are made up of low power. It works on 2.4 to $2.48 \mathrm{GHz}$ for extremely embedded sensor network. Because of $3 \mathrm{G}$ mote characteristics, it always uses higher range and larger data rate.

3. Related Research Work. In [3], the authors have presented an evaluation of performance using AODV and DYMO protocols based on CBR network using AEED, Jitter, TPR and throughput. When compared with DYMO, AODV has better throughput and less jitter and received more number of packets. In [4], authors have proposed a vigorous protocol i.e. Distributed X-layer Faster Path (DXFP) that depends on the cross layer metric which has several parameters like topology, mobility, congestion of nodes, fading, channel quality and power constraints. These are integrated with less cost which reduces the network crossing time. The DXFP protocol is further compared with AODV and DSR and is found better which improves performance and robustness of WSN.

In [5], the authors have presented a comparison of three protocols i.e. AODV, Destination Sequence 


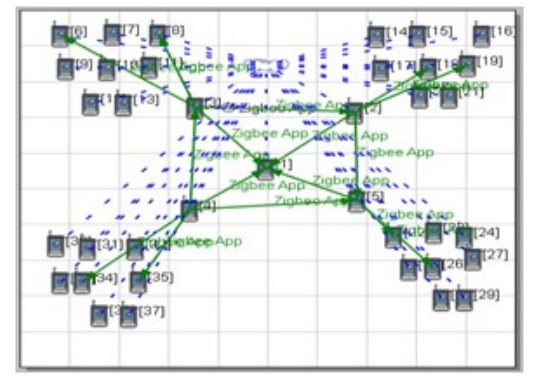

FIG. 4.1. Network Scenario I(37 static nodes) Before Simulation

Distance Vector (DSDV) and Ad-hoc on-Demand Multipath Distance vector (AOMDV) using IEEE802.11 and IEEE802.15.4 MAC standards. The simulation is done using MATLAB and NS-2 and the evaluation is based on MinMax and MinTotal performance metrics. The results determined using IEEE802.15.4 standard is greatly beneficial for engineers and designers in the energy sensitive applications and for efficient deployment of WSNs. In [6], authors have presented an approach for reducing the problem related to dropping of packets by malicious nodes by using IPSec in OLSR and STAR routing protocols in the network. From simulation outcomes, it has been evaluated that proposed approach has better TPR and throughput and has less packet dropped as compared to existing without IPSec protocol. In presence of malicious nodes, the proposed approach gives better performance of MANET.

The authors in [7] presented two reactive protocols named AODV and DYMO and then particle swarm optimization is used for optimization. For resolving the problems regarding consumption of energy and network overhead, adaptive hello messaging scheme is used and a dependable connection between the source and destination node has been created. It has been proved from the simulation results that this scheme minimised the consumption of battery and restrained needless hello messaging. In [8], the authors discussed and analyzed the Angle based routing protocols named AAODV, AOLSR and AZRP.These have been developed for Mobile Ad-Hoc networks or mobile WSNs and their comparison is done with the exiting protocols named AODV, DYMO, OLSR, ZRP. In the case if data request received, result is not greatly influenced except AAODV and in the case of transmitted signal; all the protocols are affected uniformly except AOLSR.

In [9], the authors evaluated the effects of wormhole attack using protocols DSR and AODV which has been have been analyzed and compared. After simulation, it has been observed from graphs that AODV gives better performance in case of small number of nodes but if nodes increased, overhead are also increased in huge amount. If the length of colluding link enhances, the performance for DSR performance is degraded than AODV. It is proved that, as comparison of AODV protocol, the effect of wormhole attack is much strict for DSR.

In [10], the authors have compared the three types of protocols namely: Reactive(AODV and DYMO), Proactive (OSLR) and Hybrid(IERP) protocol based upon Throughput, AEED, Jitter, PDR and TPR as performance metric using Random Way point Mobility Model. It has been concluded from simulative results that reactive protocols have improved performance incase of throughput, PDR and TPR.

In [11], the authors have evaluated OLSR and DYMO routing protocol's performance on the basis of two network metrics i.e. normalized routing load (NRL) and PDR. It has been examine that OSLR outperforms DYMO in terms of NRL and DYMO outperforms OLSR in terms of PDR. It was further observed that NRL increases and PDR decreases with increase in speed, Increasing topological area and number of nodes whereas NRL and PDR are better in case of increasing pause time and mobility speed of both protocols.

4. Simulation Setup. In this present work, we have evaluated the performance of routing protocols AODV and DYMO by using different number of nodes for a designed mobile Ad-hoc wireless sensor network with two scenarios of 37 and 50 nodes using Qualnet 7.3.1 simulator. The designed network scenarios consists of 37 nodes uniformly placed and 50 nodes randomly placed nodes in a terrain 1500 x 1500 square meters. The figures 4.1-4.4 show the designed network before and during simulation by using 37 nodes and 50 nodes separately for static and mobile scenarios. 


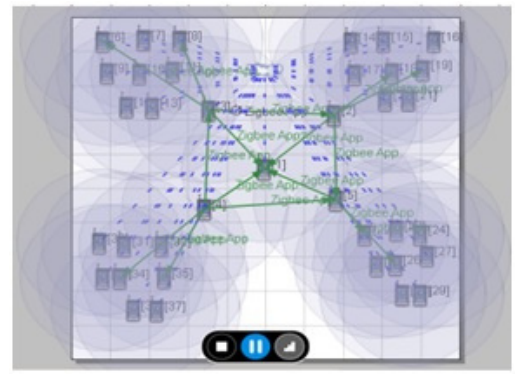

FiG. 4.2. Network Scenario I(37 static nodes) After Simulation

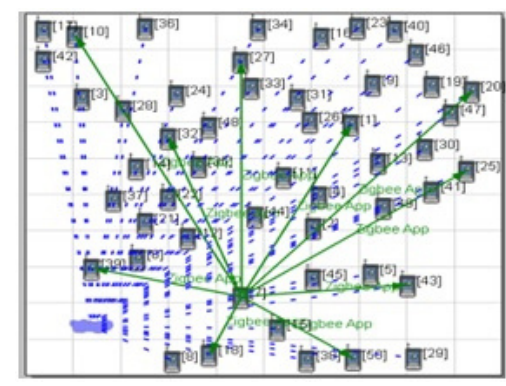

FIG. 4.3. Network Scenario II(50 static nodes) Before Simulation

For simulation, we have transmitted 100 data packets containing about 70 bytes of data over a wireless link. ZigBee application is used between the selected nodes and simulation time is kept at 500 seconds with a start and end time of 1 and 300 second respectively using "Random Way point Mobility" mobility model . The performance of three different energy conservation models Generic, Micaz and Micamotes is evaluated using AODV and DYMO routing protocol. Energy consumed in transmit and receive modes by varying number of nodes are taken as performance metric. The various parameters are considered for simulation is given in Table 4.1.

5. Results and Discussion. In this proposed work, we have simulated the designed network scenario using AODV and DYMO routing protocol in order to analyze the performance of three energy models i.e. Generic, Micaz and Micamotes.

Energy consumed in Transmit and received mode: This is equal to the total energy consumed in transmission plus reception mode. To achieve greatest sensor life, this performance metric ought to be least. Figures 5.1 and 5.2 show the variation of energy consumed in transmit and received mode for three different energy models using AODV and DYMO routing protocol for static scenario of 37 nodes.

From Figures 5.1 and 5.2, it has been observed that AODV offers enhanced performance when compared with DYMO routing protocol. Further from figure 5.1 and 5.2, the Micamotes energy model consumed lesser energy and an improved performance of $28.31 \%$ and $4.89 \%$ as compared to Generic and Micaz energy models respectively in transmit mode and an improvement of $9 \%$ and $3.5 \%$ for Generic and Micaz energy models respectively in random mobile scenario.

The Table 5.1 shows the numerical values of the graphical results of figure 5.1 and 5.2. Hence, it can be concluded that Micamotes energy model seems to be better in energy saving and the Generic model consumes more energy.

Figures 5.3 and 5.4 show the variation of energy consumed in transmit and received mode for three different energy models using AODV and DYMO routing protocol for mobile scenario of 50 mobile nodes.

From the results, it can be figured out that, behaviour of the energy consumption in transmit and received mode for scenario II is somewhat different and consumes less energy as compared to static scenario. Further from figure 8 and 9, the Micamotes energy model consumed lesser energy and an improved performance of 


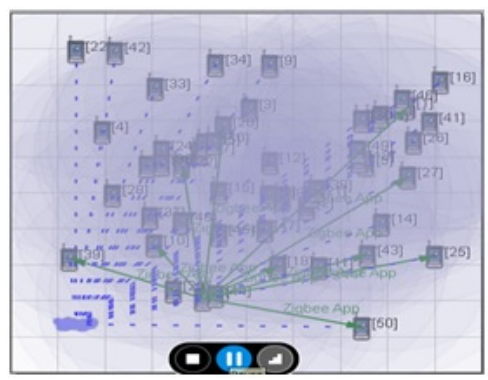

FIG. 4.4. Network Scenario II(50 static nodes) After Simulation

TABLE 4.1

Simulation Parameters

\begin{tabular}{|c|c|}
\hline Parameter & Value \\
\hline Simulator & Qualnet \\
\hline Terrain Size & $1500 \mathrm{~m}^{*} 1500 \mathrm{~m}$ \\
\hline No. of Nodes & 37,50 \\
\hline MAC Protocol & IEEE 802.15 .4 \\
\hline Packet Reception Model & PHY 802.15 .4 \\
\hline Radio Type & IEEE 802.15 .4 \\
\hline Energy Model & Generic, Micaz, Micamotes \\
\hline Routing Protocol & AODV, DYMO \\
\hline Routing Protocol & AODV, DYMO \\
\hline Traffic Type & ZIGBEE \\
\hline Link & Wireless \\
\hline Channel Frequency & 868 MHz \\
\hline Modulation & O-QPSK \\
\hline
\end{tabular}

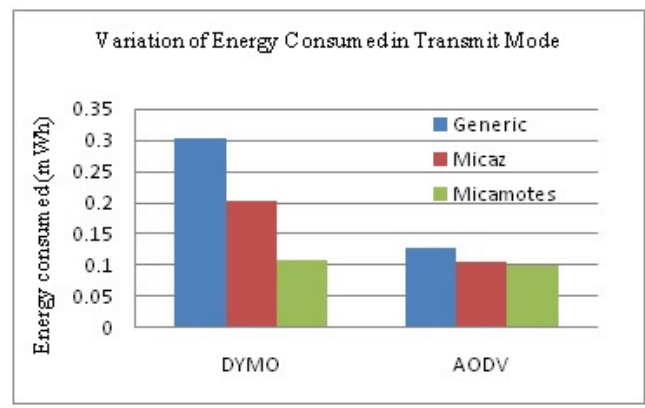

FIG. 5.1. Energy Consumed in Transmit Mode in static scenario

TABLE 5.1

Average Energy consumed in Transmit and Receive mode for static scenario

\begin{tabular}{|c|c|c|c|c|}
\hline Model & AODV Tansmit & DYMO Tansmit & AODV Receive & DYMO Receive \\
\hline \hline Generic & 0.3018 & 0.1287 & 1.755 & 0.549 \\
\hline Micaz & 0.2033 & 0.1053 & 1.249 & 0.520 \\
\hline Micamotes & 0.1073 & 0.1003 & 0.617 & 0.502 \\
\hline
\end{tabular}




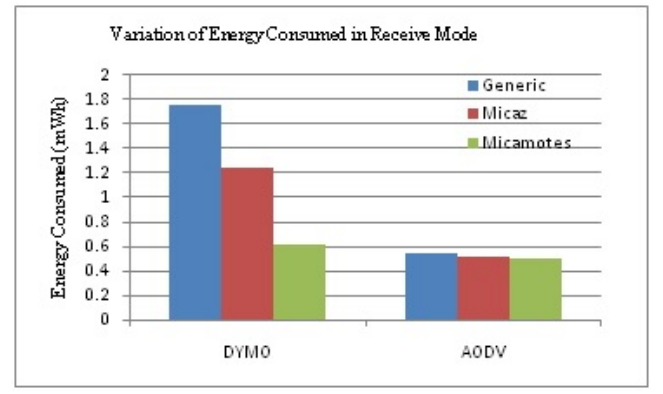

FIG. 5.2. Energy Consumed in Received Mode in static scenario

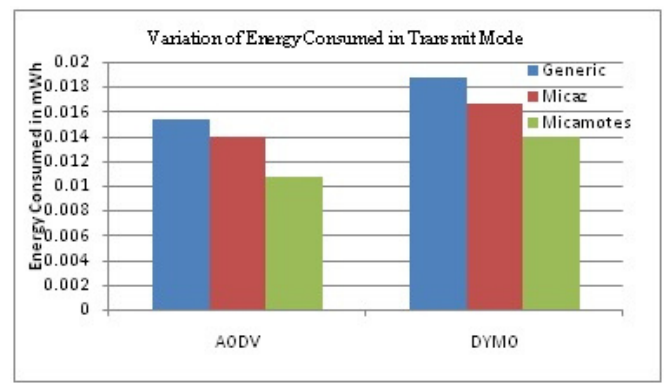

FiG. 5.3. Energy Consumed in Transmit Mode in mobile scenario

42.99\% and $29.90 \%$ as compared to Generic and Micaz energy models respectively in transmit mode and an improvement of $59.4 \%$ and $33.96 \%$ for Generic and Micaz energy models respectively in random mobile scenario. The Table 3 shows the numerical values of the graphical results of Figures 5.3 and 5.4.

Hence from Figures 5.1, 5.2, 5.3 and 5.4 and Table 5.1 and 5.2, it can be concluded that Micamotes energy model seems to be better energy saving and the Generic model consumes more energy. Further using Micamotes as energy conservation model we have carried out performance evaluation of AODV and DYMO routing protocols for the parameters (i) AEED (ii) Throughput.

$A E E D$. It is average time taken for messages to travel through the network from sender to destination. However, time interval is because of propagation, buffering, queuing and retransmissions of all existing data packets which are being sent.

From Figure 5.5, it concluded that in terms of AEED, AODV performs better and provides less AEED as compared to DYMO for both scenario I (static nodes) and scenario II (mobile nodes).

Throughput. It is the average date rate successfully transmitted over a communication channel and is measured in (i) Bits/sec (ii) Packets/sec. For a better performance, higher throughput is always desirable. The throughput variation with and without number of nodes mobility is shown in Figure 5.6.

From Figure 5.6, it can be concluded that for static and dynamic scenarios, AODV provides higher throughput as compared to DYMO protocol. Hence, from the results AODV outperforms DYMO protocol in selected performance metrics.

6. Conclusion. The performance of various energy models such as Generic, Micaz and Micamotes are analysed on the basis of network metrics such as Energy consumed in transmit and received mode over two scenarios of static nodes and random nodes using AODV and DYMO routing protocols. Form simulation results, it has been observed that Micamotes energy model using AODV routing protocol gives superior performance in terms of energy consumption upto $42.99 \%$ and $29.90 \%$ in transmit and upto $59.24 \%$ and $33.96 \%$ in receive mode respectively as compared to Generic and Micaz energy model. Upon further performance evaluation, AODV outperforms DYMO in terms of Throughput and AEED. 


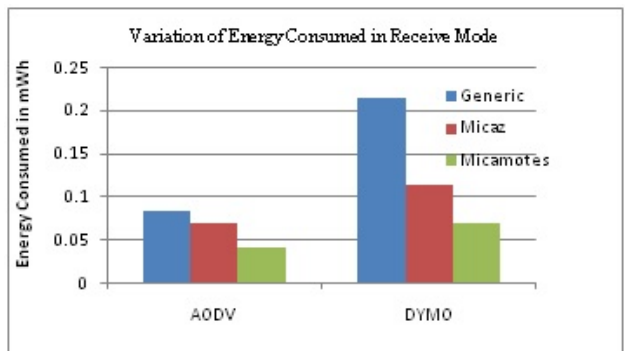

FIG. 5.4. Energy Consumed in Received Mode in mobile scenario

TABLE 5.2

Average Energy consumed in Transmit and Receive mode in mobile scenario

\begin{tabular}{|c|c|c|c|c|}
\hline Model & AODV Tansmit & DYMO Tansmit & AODV Receive & DYMO Receive \\
\hline \hline Generic & 0.0153 & 0.0187 & 0.0844 & 0.21499 \\
\hline Micaz & 0.0139 & 0.01655 & 0.0710 & 0.11409 \\
\hline Micamotes & 0.0107 & 0.0140 & 0.0530 & 0.07102 \\
\hline
\end{tabular}

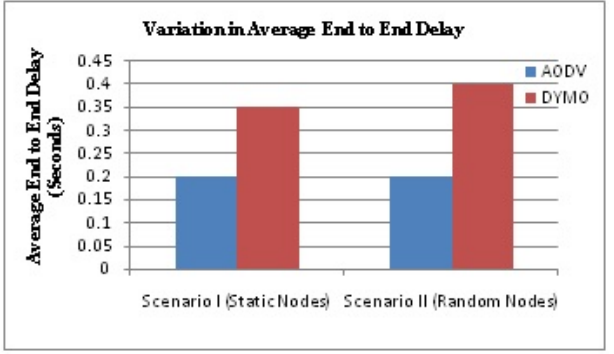

FIG. 5.5. Variation of $A E E D$ for $A O D V$ and $D Y M O$

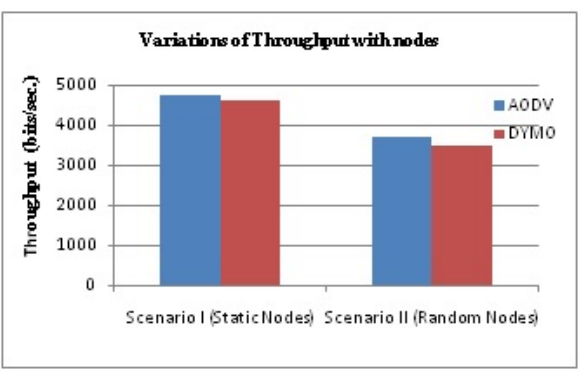

FIG. 5.6. Evaluation of Throughput(i) AODV (ii) DYMO 


\section{REFERENCES}

[1] Kirti Mor, Suresh Kumar, Deepak Sharma, Ad-Hoc Wireless Sensor Network Based on IEEE 802.15.4: Theoretical ReviewInternational Journal of Computer Sciences and Engineering. Vol.6. Issue 3, pp (219-224) March 2018. ISSN 2347-2693.doi: 10.26438/ijcse/v6i3.219224.

[2] Deepak Sharma, An overview of Wireless Sensor Networks, International Journal of Enhanced Research in Management and Computer Applications, Vol. 4 Issue 4,pp(47-51), April-2015, ISSN: 2319-7471.

[3] Payal, Deepak Sharma, Suresh Kumar, , Performance Evaluation of Reactive Routing Protocols Using IEEE 802.15.4 Application in Designed Wireless Sensor Network, International Journal of Computer Sciences and Engineering. Vol.6. Issue 4, pp (90-96), March 2018, ISSN 2347-2693.doi: 10.26438/ijcse/v6i4.9096.

[4] L. Mucchi, L. Chisci, G. Giovannettiand L. Fabbrini,, Robust Cross-Layer Routing Protocol for Mobile Ad Hoc Networks, IEEE, pp (278-284), 2012, ISSN: 4673-2569.

[5] B.Brahma Reddy, K.Kishan Rao, LU-Energy Optimization using Cross-Layer Protocols in Wireless Sensor Networks, International Journal of Computer Applications, Vol. 65- No.4, pp (18-22), March 2013, ISSN:0975 - 888.

[6] Harish Shakywar, Sanjeev Sharma And Santoh Sahu, Securing OLSR and STAR Routing Protocols against Packet Dropping by Malicious Nodes, International Journal of Computer Applications, Vol. 35- No.3, pp (7-12) , December 2011, ISSN: $0975-8887$.

[7] Sukhvinder Kaur, Sheenam Malhotra, Neighbor Discovery based on Adaptive Hello Messaging Scheme On-Demand MANET Routing Protocols using PSO, International Journal of Computer Applications, Vol. 138 - No.6, pp (36-39), March 2016, ISSN: 0975 - 8887.

[8] Saurabh mishra, Sandip Vijay, Colparative Analysis of Angle Based and Traditional Routing Protocols for MANETs / WSNs, Intelligent and Computing in Engineering, Vol. 10, pp. (251-254), 2017,ISSN 2300-5963, DOI: 10.15439/2017R90.

[9] Richa Agrawal, Rajeev Tripathi, Sudarshan Tiwari, Performance Evaluation and Comparison of AODV and DSR Under Adversarial Environment, International Conference on Computational Intelligence and Communication Systems, pp 596-600, 2011, ISSN: 7695-4587, DOI 10.1109/CICN.2011.129.

[10] Ashish Allen Roberts, Rajeev Paulus, A.K. Jaiswal, WSN Performance Parameters of AODV, DYMO, OLSR and IERP in RWP Mobility Model through Qualnet, International Journal of Computer Applications, Vol. 65- No.22, pp (28-32), March 2013, ISSN: 0975 - 8887.

[11] Muhammad Amin, Abdusalam, Abdus Salam Awan, Arshad Iqbal, Analyses of OLSR and DYMO Routing Protocols Using Normalize Routing Load and Packet Delivery Ratio in Mobile Ad-Hoc Networks, WULFENIA Journal, Vol. 19, No. 9, pp (416-437), Sep 2012, ISSN: 1561-882X.

Edited by: Ashish Kumar Luhach

Received: Jun 19, 2019

Accepted: Aug 13, 2019 